\title{
EDUCACIÓN Y DIDÁCTICA EN EL CONTEXTO DEL SABER ADMINISTRATIVO DE LOS PROGRAMAS DE ADMINISTRACIÓN LA FORMACIÓN DEL ADMINISTRADOR
}

\section{DIDACTICAL AND EDUCATION IN THE CONTEXT OF THE ADMINISTRATIVE KNOWLEDGE OF THE ADMINISTRATION PROGRAMS THE FORMATION OF THE ADMINISTRATOR}

\begin{tabular}{|c|}
\hline $\begin{array}{l}\text { Dr. Iván Darío Toro Jaramillo }{ }^{1} \text {, Jesús Ignacio Roldán } R^{2} \text {, } \\
\text { Blanca Yenny Hernández } S^{3} \text {. Claudia Patricia Vélez Y., } \\
4 \text {, Luis Fernando Agudelo } P^{5} \text {, Jorge Mario Baena, Jairo } \\
\text {, Juan Guillermo Saldarriaga }{ }^{7} \text {, Luis Alfonso Restrepo } P^{8} \text {, } \\
\text { Adriana Ramírez Cardona }{ }^{9} \text {, César Augusto Contreras }{ }^{10} \text {, }\end{array}$ \\
\hline
\end{tabular}

\section{Resumen}

Este artículo busca relacionar los conceptos de educación y didáctica con el concepto de formación. La pregunta que hoy tenemos que hacernos en el contexto de la universidad es: ¿La universidad actual está formando? Frente a tantas preocupaciones que tiene hoy la universidad el problema es que ya no se reflexiona en el acto pedagógico, sino en cómo mostrar resultados cuantificables y posicionarse como marca, lo que ha hecho que las instituciones ingresan a la lógica mercantil. En este texto se busca vincular la educación con la didáctica y sus relaciones con el saber administrativo y los programas de Administración, advirtiendo que las otras dimensiones, la pedagogía y la enseñanza, no se tendrán en cuenta de manera explícita en el estudio. Se presentan, además, algunas reflexiones de los autores consultados sobre nuevas posibilidades de aprendizajeenseñanza/educación-didáctica de la Administración, a partir de un enfoque nuevo y más coherente con el objeto de estudio de la misma: las organizaciones.

Palabras clave: educación, didáctica, administración, formación, universidad.

\footnotetext{
${ }^{1}$ Coordinador Proyecto de Investigación ASCOLFA Universidad de Medellín, Facultad de Ciencias Económicas y Administrativas, idtoro@udem.edu.co / itorojar@gmail.com.

${ }^{2}$ Egresado Universidad EAFIT, coordinador académico del proyecto de investigación

${ }^{3}$ Universidad de Medellín

${ }^{4}$ Universidad Pontificia Bolivariana

${ }^{5}$ Universidad CEIPA

${ }^{6}$ Institución Universitaria ESUMER

${ }^{7}$ FUNDACIÓN UNIVERSITARIA Luis Amigó

${ }^{8}$ Universidad de Antioquia

${ }^{9}$ Universidad de Manizales

${ }^{10}$ Universidad Nacional de Colombia-Sede Manizales

${ }^{11}$ Politécnico Jaime Isaza Cadavid

${ }^{12}$ Universidad Cooperativa de Colombia

${ }^{13}$ Universidad EAFIT y Universidad UNAULA

${ }^{14}$ Universidad UNAULA

${ }^{15}$ Universidad UNAULA

${ }^{16}$ Universidad Adventista.
} 


\begin{abstract}
This article wants to relate the concepts of education and didactics with the formation concept. The question that we have to ask ourselves in the university context is, Is the current university really forming? Before the many worries that the university faces nowadays, the problem is that we do not reflect any more on the pedagogical action as much as on how to show quantitative results and to make it position itself as a brand, As a result of that fact, the institutions have entered into the mercantile logic. This text seeks to link education and didactics and their relationship with the administrative knowledge and administration programs, clarifying that other dimensions such as the pedagogy and teaching, also will be covered in the study since it is only possible to distinguish them but do not separate them.Some reflections about the consulted authors will be presented as well. They will be about the learning-teaching education-didactics in the administration, beginning with a new focus and it will be more coherent with the object of its study: The organizations
\end{abstract}

Key words: education, didactics, administration, formation, university

\title{
Introducción
}

El concepto clave que define la educación y justifica toda didáctica debe seguir siendo la formación. En la universidad la reflexión no puede reemplazarse por la productividad. Ya no se reflexiona en el acto pedagógico sino en cómo mostrar resultados cuantificables: cuántas publicaciones se tienen, cuántos grupos de investigación, cuántos docentes, cuánta tecnología, cuántos libros y bases de datos tiene la biblioteca. La perspectiva de la cuantificación supera la reflexión y en ocasiones no se pregunta por la calidad de lo que se tiene. Las instituciones ingresan a la lógica mercantil: se venden productos académicos a través de portafolios de servicios. Así el estudiante pierde su condición de discípulo y se convierte en un "cliente" al que es necesario "cuidar", darle la razón y a quien hay que satisfacer en sus necesidades y deseos. La universidad es una empresa que busca posicionarse como marca reconocida y deseada en el mercado de la educación universitaria. En este contexto, los procesos de acreditación corren el riesgo de transformarse en medios de la estrategia comercial y de mercadeo.

En la mayoría de las instituciones educativas prima lo administrativo por encima de lo académico; se piensa que el orden al que puede llegarse por la definición de procesos y la estandarización debe estar por encima del des-orden, propio de la reflexión y la investigación; prevalece la concepción taylorista del trabajo en la que el hombre es una máquina, desconociendo las concepciones de hombre como ser biológico, sociológico, psicológico, religioso, que deben estar presentes al momento de pensar la educación, en cuanto que el hombre abarca todas esas dimensiones. También ha sido desafortunada la introducción del concepto de competencias a la universidad, cuando solo se preocupa por formar para la vida profesional y el trabajo, pues lo específico de la universidad es la discusión, la 
reflexión, la investigación y el pensar. Finalmente, el inusitado afán por la medición (medición de grupos de investigación, medición de resultados), la necesidad de mostrar indicadores de productividad y alcanzar los mejores ranking, desde uno de los principios de la racionalidad administrativa, sustento de los procesos de gestión de la calidad: "En Dios confiamos, los demás deben presentar hechos y datos. Por ello, lo que no se mide, no se administra; lo que no se administra no se mejora" (Serna, 2001, p. 7), no permite reflexionar en la educación y en la didáctica, como tampoco en la formación.

En la formación del sujeto se encuentran y articulan varias dimensiones, o campos del conocimiento: pedagogía, educación, enseñanza y didáctica, existiendo una cierta confusión conceptual en el empleo que se hace de cada uno de los términos. En este texto se busca vincular la educación con la didáctica y sus relaciones con el saber administrativo y los programas de Administración, advirtiendo que las otras dimensiones, la pedagogía y la enseñanza, no estarán presentes de manera explícita en el estudio, aunque se trate de dimensiones que sólo es posible distinguir pero no separar.

El saber administrativo ha estado plagado de teorías prescriptivas (López, 1998), que en muchos casos resultan ser "modas" que la mayoría de las veces se presentan como "la última gran salvación", que distan de establecer un corpus teórico construido desde la investigación académica. En este texto se reflexionará sobre la educación y la didáctica predominante y la formación para, finalmente, presentar algunas reflexiones de los autores consultados sobre nuevas posibilidades de aprendizaje-enseñanza/educación-didáctica de esta disciplina, a partir de un enfoque nuevo y más coherente con el objeto de estudio de la misma: las organizaciones.

En general, la práctica docente universitaria no ha considerado necesario establecerse en el saber de la educación ni en el de la didáctica, pues "en su lugar se requieren docentes con desarrollos académicos y científicos en un área determinada del saber. Un indicio de ello es que para seleccionar a los profesores universitarios no es un requisito la formación en pedagogía, ni en didáctica, más bien es indispensable la formación como investigadores" (Grisales y González, 2009, p.78). Las universidades a partir de estándares de calidad que se les impone, exigen a sus docentes la formación postgraduada, el manejo de otras lenguas, ser investigadores y tener publicaciones académicas, y para que éstas valgan deben hacerse en publicaciones indexadas o de alto impacto.

Tales planteamientos entrañan a la vez que una dificultad también una limitación, toda vez que hoy no es fácil comprender lo que significa y el alcance que tiene lo que Comenio pedía para la academia: contar con profesores "sabios y eruditos" (Grisales y González, 2009, p. 79), y que, además, todo este conocimiento sea resultado de su propia actividad científica. Asimismo, se abre la posibilidad de distinguir el campo de acción del investigador y el del profesor: no se discute acerca de la importancia y la necesidad de la investigación para la misma actividad o ejercicio docente, pero lo que no se puede aceptar es que el 
docente/profesor pierda el lugar que siempre ha tenido en la educación y la didáctica, el papel de ser profesor como tal, o en cuanto tal. Lo que llevaría a seguir percibiendo al profesor no sólo como una persona poseedora de un saber (erudito), sino como alguien que se considera sabio.

En términos de antecedentes, y desde la postura de Comenio, citado por Grisales y González (2009),lo que realmente importa hoy en la docencia universitaria es el contenido de lo que se enseña, esto es, el contenido de las ciencias, y que se realice de manera erudita, toda vez que se concibe al docente como un digno exponente, como un depositario vivo del conocimiento, o mejor, como un sabio que produce el contenido de las ciencias que enseña, lo que nos lleva a entender que en la selección de dichos docentes tenga mayor relevancia la formación como investigadores, que como docentes propiamente dichos, lo que permitiría concluir, igualmente, que para enseñar es suficiente con saber y con producir conocimiento. Pensando en la enseñanza y la didáctica habrá que reconocer no sólo lo que es necesario aprender, para definir lo que se necesita enseñar, sino también cómo llegar a la formación.

\section{Aspectos de la metodología}

Este texto es el resultado de un largo trabajo que se desprende del proyecto de investigación que investigadores de 13 universidades que vienen realizando desde hace dos años (2010-2012), con el propósito de hacer un aporte a la educación y a la didáctica en relación con el saber administrativo. Una anterior investigación a esta, acerca de la situación de la investigación en los programas de Administración, arrojó como resultado que la mayor preocupación de nuestras escuelas y programas de Administración no estaba sólo en el asunto de la investigación y los grupos de investigación, sino principalmente en la formación de los docentes en campos como la educación y la didáctica. Fue esta la razón que tuvo la Asociación Colombiana de Facultades de Administración (AsCOLFA), de pedir a un grupo de investigadores la realización de una investigación a partir de estos temas de la educación y la didáctica.

El punto de partida de esta investigación estuvo en la pregunta: desde la Administración, ¿cómo contribuye al logro de los objetivos de formación la relación entre educación y didáctica? Dicha pregunta respondía, a su vez, a una serie de preocupaciones que han sido discutidas por el grupo de investigadores, como, por ejemplo: ¿Qué es lo que sucede en el aula?, ¿qué tipo de docentes requieren nuestras universidades, con qué formación y qué se les debe exigir?, ¿cómo preparar a los docentes en relación con el acto pedagógico?, lo que también implicaba hacer preguntas acerca de la formación que se está dando en la universidad y el tipo de estudiantes que se tienen en nuestras escuelas y programas de Administración (¿clientes o estudiantes?).

Lo anterior hizo que el grupo de investigación se formulara una serie de objetivos que orientaran la investigación, como fueron, por ejemplo, el buscar analizar la relación entre educación y didáctica, para determinar su contribución al logro de los objetivos de formación y la comprensión de la disciplina administrativa, a través 
de la vinculación de las facultades de Administración con la investigación. Lo que llevó también al grupo de investigación a tener que definir los conceptos de educación y didáctica que sean más pertinentes con la disciplina administrativa, a partir de la construcción de un estado del arte, que ayudara a comprender mejor la relación entre dichos conceptos. Además, se buscó caracterizar las didácticas presentes en el aula que ayuden a conocer el proceso educativo que se está realizando en las facultades o programas de Administración, a través de un trabajo de campo. Finalmente, el grupo de investigación ha tenido como preocupación el poder identificar las actuales tendencias y perspectivas de la educación y la didáctica y las nuevas condiciones (globalización, regulación, TIC, mercado laboral) que inciden en el campo de la disciplina administrativa en Colombia.

La investigación completa recorre tres etapas. Este artículo recoge los resultados de la primera etapa, de carácter exploratorio, que sirvió para elaborar un estado del arte en relación con los conceptos de educación y didáctica que fueran más pertinentes con la Administración. Las etapas siguientes corresponden al trabajo de campo (encuesta a una muestra significativa de estudiantes de las escuelas y programas de Administración, principalmente de Antioquia, entrevistas en profundidad con directivos y grupos focales con docentesque puedan dar cuenta del estado actual y de las perspectivas futuras de la formación de los administradores), y, finalmente, la sistematización de la información obtenida a lo largo de toda la investigación.

\section{Aspectos fundamentales acerca de la educación}

Sin lugar a dudas, el término más amplio corresponde al de educación, entendida como el proceso de socialización del hombre. La educación es un acontecimiento típicamente humano, un proceso intencional, un logro cultural, una práctica social. En otras palabras, la educación es un desarrollo planeado e intencional. Lo intencional está en la misma definición etimológica de la palabra educación (Lucio, 1989).

El hombre siempre está en continuo crecimiento y lo hace en un entorno social. "Educación, en sentido amplio, es el proceso por el cual la sociedad facilita, de una manera intencional o difusa, este crecimiento en sus miembros. Por tanto, la educación es ante todo una práctica social, que responde a, o lleva implícita, una determinada visión del hombre (de lo que se puede llamar su "crecer")" (Lucio, 1989, p. 1).

Referirse a la educación atañe a lo que por siglos se ha elaborado como la teoría de la educación, que tiene como máximo referente lo humano, en cuanto a las capacidades propias de su biología; como de su condición individual (su psiquis), social (en relación con los otros y lo otro) e histórico-cultural (su necesidad y capacidad de transmitir, dejando en la memoria, sus adquisiciones/conquistas tecnológicas, teóricas y simbólicas). 
Educar es formar, la educación conduce a la formación, lo que conlleva a pensar en el desarrollo e intencionalidad, tanto en lo individual como en lo social. A partir de los distintos autores que tratan el tema de la educación surgen múltiples interpretación de los que el concepto significa y acerca de cuáles pueden ser sus implicaciones.

Paciano Fermoso en su teoría de la educación, desde una interpretación antropológica, define la educación como un "proceso exclusivamente humano, intencional, inter-comunicativo, y espiritual, en virtud del cual se realizan con mayor plenitud la instrucción, la personalización, la socialización y la moralización del hombre" (1985, p.162).

Para Patiño y Rojas (2009), la educación se desarrolla en un campo de contradicciones: está entre el disciplinamiento y la individualización. Las actividades en el aula permiten comprender complejos procesos de transformación o afirmación de subjetividades, los cuales se encuentran mediados por las relaciones con nosotros mismos, con los otros y con el conocimiento.

También la educación se ha creído que debe estar sometida a ciertas exigencias relacionadas con la pertinencia, calidad, cobertura, cuando se entiende que la educación debe responder a las tendencias políticas, económicas y culturales, exigiendo nuevas formas de interpretar y desenvolverse en el mundo o el contexto que la rodea. Se piensa, aunque de manera no muy acertada, que tal y como cambian las formas de trabajo, de producción, y las demandas de conocimiento también cambia la educación, como cuando se piensa en la relación universidadempresa, de modo que la universidad pueda dar respuesta a dichas demandas, además de aquellos otros retos que hacen parte de su esencia: como la construcción de conocimiento y el entendimiento y comprensión del individuo como ser humano y parte de una sociedad.

Delors (1998), considera que la educación debe estructurarse en torno a cuatro pilares, a los cuales subyace el conocimiento: aprender a conocer (comprensión), aprender a hacer (influencia en el entorno), aprender a vivir juntos (cooperaciónfraternidad) y aprender a ser (autonomía-juicio-responsabilidad). Asimismo, en este orden, Lee Shulman (2005 )identifica tres aspectos críticos y fundamentales en la formación profesional: pensar, realizar y actuar con integridad, aunque, como lo señala este autor, estas tres dimensiones no reciben igual atención a través de las profesiones.

Debe entenderse que el profesional no sólo se forma para entender los cuerpos de conocimiento que nutren una determinada área del saber, sino que además esta preparación está al servicio de otros y por ello la importancia de educarse para un "buen trabajo" (Shulman, 2005).No obstante, de otro lado, también están las exigencias de una sociedad de consumo en la que prima la producción de mercancías, que le exige al hombre tener las destrezas necesarias para producir eficientemente, para lo cual resulta necesaria la información y acumulación de datos y conocimientos, que permitan ser más "eficientes" en la producción de 
bienes y servicios, como si hubiese una época para educarse y otra diferente para producir, como diría Lucio (1989), haciendo que la educación no sea un hecho permanente de crecimiento.

Este tipo de reflexiones en torno a los escenarios en los que se desenvuelve la educación, donde se presentan problemas relativos al conocimiento, a la práctica y a sus interrelaciones, conducen necesariamente a hablar del concepto de pedagogía o ciencia de la educación, como la denomina Vargas Guillén.La educación es una alternativa clara que permite la potencialización del individuo y el desarrollo de las comunidades: "La educación tiene la misión de permitir a todos sin excepción hacer fructificar todos sus talentos y todas sus capacidades de creación, lo que implica que cada uno pueda responsabilizarse de sí mismo y realizar su proyecto personal" (Delors, 1998, p. 14).

El proceso educativo está permeado por muchas prácticas de tipo pedagógico, metodológico, didáctico, entre otras, que han sido heredadas de las diferentes corrientes educativas que han permanecido a lo largo del tiempo, pero también están influenciadas por las nuevas prácticas, por aquellas corrientes activas y transformadoras, que se suponen se adaptan mejor al contexto actual y a las necesidades de las generaciones de estudiantes que en la actualidad se están formando en la universidad.

Las facultades de Administración no son ajenas a dichas prácticas educativas y su proceso de construcción educativo y del conocimiento se ha visto impactado por una larga carrera que ha buscado que la Administración, así como las ciencias sociales en general, encuentre su lugar en relación con su método y con el conocimiento, en relación con el mundo de las ciencias exactas, las cuales indiscutiblemente ya tienen una serie de desarrollos más claros al respecto.

\section{Aspectos fundamentales acerca de la didáctica}

En la relación entre la enseñanza y la didáctica, surge la pregunta por el ¿cómo enseñar?, hay que saber cómo se aprende (diseño curricular, psicología del aprendizaje). La concepción tradicional de la didáctica la concibe como un espacio de concreción normativa para la realización de la enseñanza. En el siglo XIX se enfatizó en el proceso de la instrucción como transmisión del saber y el discurso de la didáctica estuvo ligado a la búsqueda de utopías pedagógicas, la concreción de reglas de acción para la enseñanza, conforme a pasos y medios determinados, el desarrollo de los sistemas educativos modernos y el optimismo pedagógico respecto de la construcción de la sociedad más justa y humana.

Para De Mattos (1983) la didáctica tradicional procuraba responder a los siguientes interrogantes: ¿A quién se enseña?, ¿quién enseña?, ¿para qué se enseña?, ¿qué se enseña?, ¿cómo se enseña?; mientras que la didáctica moderna pretende responder a los siguientes interrogantes:¿Quién aprende?, ¿con quién aprende el alumno?, ¿para qué aprende el alumno?, ¿qué aprende el alumno? y ¿cómo aprende el alumno? Desde dicha perspectiva en el proceso 
didáctico propuesto por el autor intervienen dos binomios fundamentales. El primero corresponde al binomio humano conformado por el maestro, los alumnos y las interacciones entre ellos, y el segundo binomio es el cultural, conformado por la materia y el método al servicio del binomio humano y para responder a los objetivos propuestos. Para De Mattos, el segundo binomio, denominado materia y método, cumple un papel de apoyo y soporte al binomio principal (humano), por lo tanto no se debe perder de vista al binomio humano por el binomio secundario.

El siglo Xx marcó un nuevo enfoque en el desarrollo: la expresión de la escolaridad pública, el liberalismo político y los avances en el conocimiento de la psicología del desarrollo facilitaron la concreción de experiencias significativas en el campo didáctico. Es así como aparece un relativo desplazamiento desde el disciplinamiento y la instrucción hacia el desarrollo del sujeto del aprendizaje. Se encuentran expresiones como las de Montessori, los centros de interés de Decroly, los postulados de Ferriere, la escuela de trabajo colectivo de Freinet, entre otros, (Camilloni, et al., 1996, pp. 45-46).

Se tienen actualmente discursos que se desprenden de la hermenéutica, desde donde se ha tratado de interpretar los procesos que caracterizan la vida del aula, reconstruyendo el significado y las intenciones de los individuos. Otros enfoques impulsan la investigación como componente del desarrollo del currículum y la participación directa de los docentes en el proceso (Camilloni, et al.,1996). Todas las propuestas devenidas de la escuela crítica en contraposición de la positivista tradicional, le dan un enfoque distinto a la didáctica, poniendo como centro al estudiante y la interacción simultánea docente-alumno, desde una postura que promueve la reflexión y la crítica de docentes para comprender y operar la enseñanza. Buchelli y Marín (2009) consideran que desde esta perspectiva crítica, se genera una corresponsabilidad de los estudiantes y docentes por alcanzar los propósitos; por tanto, nuevos roles deben cumplir unos y otros. Uno de esos roles y que es común a ambos, se relaciona con la investigación como una actividad permanente en su quehacer. En este contexto, la clase y el aula se convierten en la discusión dialéctica, creativa y propositiva, sobre bases de argumentación y diálogo teórico-conceptual.

Según Flores (1994), la didáctica comprende las metodologías de enseñanza, el conjunto de métodos y técnicas que permiten enseñar con "eficiencia", lo que ha hecho que en muchos casos se comprenda la didáctica como el aspecto más instrumental de la pedagogía; sin embargo, no ha de reducirse a las herramientas pedagógicas (Rozo, 2008), pues tal reducción dejaría de lado el contenido de las interacciones humanas, epistemológicas y disciplinares que se establecen entre los saberes.

Siguiendo a Vasco (1990) y a Rozo (2008), el planteamiento de la didáctica debe ser más sistémico, debe reconocer además de métodos y técnicas las múltiples y diversas relaciones del maestro con sus alumnos y sus micro-entornos desde el punto de vista de la enseñanza. Contreras (1990) considera que la relación enseñanza-aprendizaje no se presenta casualmente; existe dado que se puede 
presentar enseñanza sin que se cause necesariamente el aprendizaje, esta se encuentra necesariamente mediada por la circulación de actividades establecidas en un contexto colectivo, es decir, la didáctica se define como "disciplina que explica los procesos de enseñanza aprendizaje de acuerdo a la realización de los fines educativos" (Contreras, 1990, p. 182).

\section{Educación y didáctica en relación con el saber administrativo}

Para comprender la educación y la didáctica en el contexto de la Administración, es necesario reconocer el marco histórico, económico y social en el que surge esta disciplina y la manera como se organiza. Los desarrollos económicos a partir del siglo XIX representan fuerzas significativas e impulsoras de la educación en Administración. Barrera y Sanclemente afirman que "la administración científica emerge a comienzos del siglo $x x$ en un contexto donde predominaba la lógica positivista de la economía neoclásica y el racionalismo científico de la modernidad [...] en contextos organizacionales donde florecían el énfasis en la productividad y en la eficiencia del trabajo del obrero" (2008, p. 13).

Esta ideología gerencial que interpreta la realidad desde lo racional y lo productivo, al individuo como un medio de producción y un recurso para alcanzar la productividad y la eficiencia en función de lo económico, a la empresa como el espacio donde se logra la maximización del crecimiento, el valor económico y la optimización de los flujos de caja, lleva a la conclusión de que "[...] el origen del pensamiento administrativo fue impulsado por los dogmas ideológicos de la maximización de la utilidad y la creación de valor económico para los propietarios del capital" (De Gaulejac y Aubert, citados por Barrera y Sanclemente, 2008, p. 14).

De otra parte, Weber (1973) define que el "espíritu del capitalismo" consiste en la mentalidad que busca el lucro y su reproducción mediante el ejercicio de una profesión obteniendo de ella una ganancia legítima. Este fin de lucro que se le asigna al ejercicio de la profesión articulado a la metáfora del homo economicus cuya esencia es el egoísmo, identifica los valores que subyacen en el modelo capitalista moderno.

La educación y didáctica de la Administración definen su accionar en el mundo práctico de las empresas en las cuales se domestica la mente del estudiante en una fuerte cultura positivista, se enseña a la identificación objetiva de los problemas a través de un modelo racional de carácter universal que desestima lo cualitativo y, por último, se mitiga la importancia de la dirección de las personas a través de la expresión de un sentido ético y de respeto por la dignidad humana (Barrera y Sanclemente, 2008, p.30).

Esta implantación del positivismo técnico-racional connatural a las ciencias exactas ha penetrado la Administración como ciencia social, llevándola al uso predominante de metodologías deductivas. Refiriéndose a este tema, el proyecto 
Tuning ${ }^{17}$ expresa que los administradores en la práctica excluyen las preferencias personales y la experiencia no sistematizada de las decisiones profesionales dando prioridad a las mejores evidencias científicas disponibles.

Autores como Vance, Groves, Yongsun y Klindler (2007), plantean que el estilo de pensamiento occidental está influenciado por una visión newtoniana, determinista y reduccionista, orientada al equilibrio, la cual pretende dividir las partes del sistema, lo que se denomina "reduccionismo lineal", basado en una metodología analítica, donde se concibe el sistema igual a la suma de sus partes. Estos autores afirman que dicho sistema se queda corto para entender y abordar sistemas no lineales, complejos e impredecibles, donde el comportamiento del conjunto no es necesariamente igual a la suma de sus partes. Como modelos alternos a esta forma habitual de enseñanza en las universidades de occidente se sugiere tener en cuenta aquellas formas de conocimiento basadas en la intuición, la creatividad, la flexibilidad, la visión holística, la emoción o una combinación de las aquí mencionadas.

Obviamente, es imposible desestimar la importancia del método deductivo; sin embargo, se cuestiona su pertinencia a partir del hecho de que la Administración no es una ciencia pura y que por lo tanto no hay verdades absolutas. Para Mintzberg la dependencia sobre la deducción puede atrofiar la teoría del desarrollo, a la vez que no hace posible a los investigadores proyectarse más allá de los datos existentes (Mintzberg, 2005). Tal dependencia excesiva sobre la deducción impide a los individuos comprometerse en la "imaginación disciplinada"(Day, G. y Schoemaker, P. 2001): esencial para el desarrollo de una buena teoría.

A partir de la aplicación de un instrumento validado por Vance et al., nombrado perfil de estilo de pensamiento lineal no-lineal (sigla en inglés LNTSP) en una población diversa compuesta por cuatro muestras distribuidas así: Muestra 1: estudiantes universitarios de comercialización, ingeniería, gestión, psicología, finanzas y negocios internacionales. Muestra 2: directores de contratación de grandes empresas multinacionales, y un programa de MBA compuesto por empleados de la industria aeroespacial, defensa, telecomunicaciones y servicios financieros. Muestra 3: Estudiantes universitarios de Administración de empresas de una universidad privada de tamaño medio en el suroeste de Estados Unidos y Muestra 4: actores profesionales y contadores, los investigadores trataron de medir el estilo de pensamiento lineal y no lineal de preferencia en los participantes.

Las conclusiones a las que llegaron una vez aplicado el instrumento, plantearon, entre otros asuntos, que el estilo de pensamiento lineal se puede medir por la preferencia de un individuo para considerar datos externos y los hechos y procesamiento de la información a través del pensamiento racional para guiar la acción posterior, mientras que el estilo de pensamiento no lineal puede ser evaluado por la preferencia de un individuo para atender fuentes internas, tales

${ }^{17}$ Este proyecto fue editado en un texto para América Latina por Beneitone, et al., (2007). 
como los sentimientos, la intuición, corazonadas, para informar y orientar la acción posterior; por otro lado, presentan un dato que llama la atención y que quizá confirma una condición ya discutida en entornos de disciplinas como la psicología, la sociología y la neurología, hace referencia a los resultados que se encontraron en términos de diferencia de género y de profesión. Vance,et al., (2007), reconocen que las mujeres y los actores presentan una preferencia alta al pensamiento no lineal; por el contrario los hombres, en particular los ingenieros, contadores y personas mayores, tienden más a la toma de decisiones por medio del pensamiento lineal.

Entre las sugerencias que plantean a partir de los resultados encontrados como retos para la gestión de la educación, establecen que los profesores deben diagnosticar con precisión el estilo de pensamiento de los estudiantes, para construir estrategias que apunten a desarrollar en unos y otros el estilo de pensamiento que se encuentra más débil o menos presente, de tal suerte que los estudiantes con preferencia por el estilo de pensamiento lineal desarrollen capacidades en el estilo de pensamiento no lineal y viceversa, "(...) para lograr un modelo sinérgico que utilice los puntos fuertes de ambos estilos" (Vance,et al., 2007, p. 180).

\section{Las nuevas tecnologías en educación y sus desafíos}

Siguiendo con el interés que nos convoca en este escrito, en relación con la educación y la didáctica en el saber administrativo, encontramos otros antecedentes en estudios que se inclinan por la presencia de las nuevas tecnologías en la educación y los desafíos que las mismas presentan. Es así como Phan, Siegel y Wright (2009), Ilaman la atención sobre cómo las políticas públicas inciden en la vinculación de empresa-universidad-estado, toda vez que reconocen la necesidad de construir desarrollo tecnológico.

Respecto a las iniciativas que influyen en la política de gestión tecnológica mencionan: "Políticas de fomento de la transferencia tecnológica de universidades y laboratorios federales a las empresa. Un gran aumento en la incidencia de los actores públicos y asociaciones público-privada. Flexibilización de las normas antimonopolio relacionadas con la investigación colaborativa"(Phan, et al. 2007: 325). Plantean, entonces, la necesidad de desarrollar capacidades en los estudiantes, como el trabajo en equipo y especialmente en equipos interdisciplinarios, que permitan construir diálogos entre las distintas disciplinas sociales y técnicas, de tal suerte que logren discusiones y desarrollos tecnológicos con el apoyo de la empresa.

Con la intención de alcanzar el objetivo de lograr el desarrollo tecnológico que los autores defienden, recomiendan que los directores de las escuelas de negocio cuenten con un perfil que abarque habilidades en tecnología para facilitar vínculos fuera del campus y dentro de él.Phan,et al., (2007) entienden que existe una tendencia en las escuelas de negocios, especialmente en el Reino Unido y en Estados Unidos, a ser escuelas independientes del resto de las universidades; tratando de cerrar la brecha, recomiendan que dichas escuelas establezcan 
conexiones más fuertes con las escuelas de ingeniería, ciencias y otras organizaciones orientadas a la tecnología en las áreas de medicina, la salud pública, farmacia, así como incubadoras de empresas basadas en la ciencia y parques tecnológicos (Phan,et al., 2007, p.331).

Entre otras sugerencias para realizar la adecuada vinculación entre tecnología y educación plantean la posibilidad de construir planes de negocios establecidos apropiadamente, de tal manera que logren atraer la atención de los estudiantes a partir del aprendizaje experiencial, de tal manera que los docentes sean además mentores de sus estudiantes. La transferencia de conocimiento a partir de la movilidad del personal, permitirá que los docentes de unas y otras escuelas intercambien conocimiento en términos de tecnología; finalmente, se propone una adaptación de la pedagogía, en los mecanismos de entrega y secuenciación de contenidos, basados en el gobierno de iniciativas.

\section{La evaluación como parte de la educación y la didáctica}

Si bien las formas de enseñanza en términos de la didáctica son elementos que requieren especial atención en el sentido del problema que interesa, es importante virar hacia la evaluación de dicho proceso de aprendizaje. La educación se ha preocupado por el cómo evaluar, de tal manera que el resultado dé cuenta en realidad de lo aprendido. En este sentido se han escuchado discursos que han puesto el énfasis en la autoevaluación del conocimiento, asumiendo que el alumno está en la capacidad de evaluarse a sí mismo; sin embargo, se encuentran discursos que interrogan la efectividad de la autoevaluación misma, en tal sentido encontramos a Armstrong y Fukami (2010), quienes se preguntan si la autoevaluación del conocimiento es un aprendizaje cognitivo o medida efectiva. En realidad estos autores no defienden o refutan la autoevaluación en sí misma, lo que ponen en tela de juicio es la forma y los resultados del proceso auto evaluativo. Entre otras posturas que traen a colación expresan que la autoevaluación es más afectiva que efectiva.

Partiendo del componente afectivo que tiene la autoevaluación, se encuentra que la mayoría de las veces la autoevaluación positiva está relacionada más con la

sensación de placer o de satisfacción que con el aprendizaje mismo. Como Armstrong y Fukami (2010) plantean, no se evalúa el verdadero aprendizaje toda vez que este no necesariamente tiene que ser placentero. La psicología evolutiva establece que el proceso de aprendizaje en el tránsito por el desarrollo del ser humano es doloroso, conflictivo y tenso, no necesariamente obedece a un acto de afecto positivo (Papalia, Wendkos, Duskin, y Gross, 2005); por lo tanto, la autoevaluación cuando el aprendizaje está acompañado por los afectos antes mencionados resulta ser negativa, asumiendo que este componente no entraña aprendizaje en sí mismo. En este sentido, la autoevaluación no cumple con su real cometido: dar cuenta si el estudiante en realidad obtuvo aprendizaje.

Armstrong y Fukami (2010), citando la propuesta de Rubin y Martell (2009), expresan que para elegir métodos apropiados de evaluación se deben especificar 
los conocimientos, habilidades o comportamientos asociados con el aprendizaje de las metas y objetivos. En esa dirección establecen que el aprendizaje debe ser evaluado en términos de:

1. Lo cognitivo: los resultados del aprendizaje que se refieren a la adquisición de los conocimientos que se pueden clasificar en los tres ámbitos de: conocimiento declarativo (cantidad y exactitud de los conocimientos adquiridos), organización del conocimiento (la comprensión de las interrelaciones entre las estructuras del conocimiento) y las estrategias cognitivas (formación en conceptos y procedimientos)

2. La habilidad: resultados del aprendizaje de habilidades basadas en involucrar y demostrar habilidades técnicas o el motor que los alumnos no han declarado, así como la capacidad para realizar estas habilidades con fluidez en condiciones reales.

3. Lo afectivo: se refiere a los resultados del aprendizaje de los alumnos, actitudes y motivaciones hacia el particular objetivo de aprendizaje, incluidas las condenas de los alumnos y los niveles de confianza, también conocido como auto-eficacia (Armstrong y Fukami, 2010, p. 338).

Respecto a qué se debe evaluar en el contexto de la gestión de la educación, se pretende que se conjuguen el conocimiento declarativo (hace referencia al conocimiento consciente, formado, controlado) y el conocimiento tácito (hace referencia al conocimiento inconsciente, automático, que guía las acciones y decisiones sin estar en nuestro campo consiente), y esto además de tener implicaciones en la formación de los estudiantes para hacerse cargo responsablemente de su propia autoevaluación, tiene efectos en la modificación de enfoque de la enseñanza aprendizaje, en donde el docente se convierte en tutor de la enseñanza en un afán por comprometer al estudiante en la participación casi total de su aprendizaje y formación.

\section{Investigación y publicaciones en administración}

Centrando la discusión en torno a la gestión de la educación en la Administración, particularmente algunos autores han preguntado por el liderazgo y la administración de la educación superior (Taylor y Machado, 2006) y la educación administrativa crítica (Learmonth, 2007). Este último particulariza el problema a la formación de los administradores y expresa que el eclecticismo presente ha servido para esconder un hilo importante de continuidad dentro de sus ideas y teorías dominantes. Por otro lado, y como ya se había expresado antes en este escrito, en la práctica educativa actual de la Administración existe una preferencia por el conocimiento deductivo más que por el inductivo. Esta preferencia metodológica contrasta con la declaración de que la Administración no es una "ciencia pura", por lo tanto no existen verdades verificables y se invalida lo que se denomina como una "administración basada en evidencias".

Mintzberg (2005), como muchos otros autores que hacen serias críticas a los programas de MBA, afirma que los programas actuales de formación en postgrado en Administración, los MBA, se enfocan la mayoría de las veces en las 
"funciones de negocio" y no en la práctica de la administración. Lo que genera una desconexión entre lo que es enseñado y lo que se necesita para ser un administrador efectivo. De otra parte, lo que es enseñado está fuertemente influenciado por lo que investigan y escriben los docentes de Administración, investigaciones que ponen en evidencia la fuerte desconexión que existe con las características de la administración diaria.

Se presenta entonces como indicador a los elementos antes puestos en discusión, el hecho de que solo tres de los libros más vendidos en Administración corresponden a académicos. Learmonth (2007) atribuye este fenómeno a que posiblemente esta situación pone énfasis en el conocimiento deductivo sobre el inductivo. En este orden de ideas y respecto a publicaciones en tres de las más reconocidas revista en pedagogía en EEUU, a saber: Journal of Management Education (JME), Academy of Management LearningandEducation (AMLE) y Management Learning (ML), Beatty y Leing (2010), mencionan que la hegemonía sigue siendo tanto en edición como en investigación Gran Bretaña y Norte América, allí se encuentran reunidos la gran mayoría de investigadores que publican en estos medios, reafirmando con ello el paradigma occidental.

De otro lado, en función de los problemas que reconoce la Administración, están las diferentes concepciones de la educación y sus propuestas pedagógicas. Una manera de identificarlas es desde la revisión documental realizada por las profesores KirsiKorpiaho, HannaPäiviö y KeijoRäsänen (2007), bajo la perspectiva teórico-práctica, de las tres principales revistas angloamericanas en investigación sobre educación en Administración, como lo son: TheJournal of Management Education, TheAcademy of Management LearningandEducation y Management Learning. A partir de este trabajo pueden identificarse seis concepciones de la educación en Administración, que incluyen la perspectiva tradicional e introducen como alternativas otras cinco concepciones.

La educación tradicional se enmarca organizada de acuerdo con los problemas funcionales de la Administración y las disciplinas como el comportamiento organizacional, el mercadeo, la contabilidad y finanzas. El profesor se caracteriza por ser un especialista en su área o sub-área; es un experto que enseña instrumentos para la práctica más que aprendizaje o desafíos intelectuales (Korpiaho, et al., 2007). Con ello el estudiante termina dotado de un amplio conjunto de técnicas administrativas, entregadas a través del currículo que abarca las sub-disciplinas de mayor utilidad para el profesional, dando paso a la especialización en varias funciones o sub-tareas administrativas (Korpiaho, et al., 2007). Esto se imparte haciendo uso de modalidades de enseñanza como la clase "magistral", el estudio de casos y medios informáticos.

La educación tradicional o la corriente principal en Administración representa el eje de la crítica, básicamente en torno a sus cuestionamientos éticos y morales, desprendidos por la alta especialización, fragmentación en los contenidos, reducción de la realidad y la promesa de dotar de instrumentos, a manera de 
fórmulas útiles y efectivas, a cualquier tipo de organización, condiciones y circunstancias.

Se originan cinco concepciones adicionales a la educación tradicional: educación basada en la ciencia, educación basada en las competencias, las formas alternativas de educación -aprendizaje basado en el servicio (service-learning)-, educación basada en la acción y la educación crítica de la administración (Korpiaho, et al., 2007).

La educación basada en la ciencia se fundamenta en la relevancia otorgada a la investigación científica y responde a la acusación de la falta de credibilidad académica de los programas (Korpiaho, et al., 2007). Siendo así, se reclama que tanto estudiantes como docentes deben beneficiarse del conocimiento producto de la investigación y así el objetivo es darle un nivel superior a la Administración como profesión. No obstante, según el estudio de Korpiaho, Päiviö y Räsänen, esta aproximación basada en la ciencia ha privilegiado la producción de "conocimiento científico" y no así las preocupaciones por los asuntos morales (2007, p.47).

La educación basada en las competencias surge como respuesta a la crítica de que los administradores formados sólo en el conocimiento científico fracasan al emprender trabajos administrativos con estándares aceptables de desempeño. Esencialmente este modelo implica definir y categorizar la plataforma de las competencias relevantes y de allí los objetivos de formación del estudiante (Korpiaho, et al., 2007). El vacio que se detecta es que en la integración de la habilidad con la herramienta no se incluye la reflexión crítica, por eso al comienzo de este artículo señalábamos el desacierto al haber introducido el asunto de las competencias en la universidad.

Las formas alternativas de educación en términos generales se alejan de las aproximaciones tradicionales y tratan de poner en contexto los agentes de la educación en Administración, de tal forma que la vinculación de otras alternativas de formación cobran sentido, se aboga por una mayor disposición hacia prácticas más críticas, el compromiso hacia nuevos modelos y paradigmas (Ashkasasy, citado por Korpiaho, et al., 2007). El aprendizaje basado en el servicio (servicelearning) es propuesto para la educación en Administración en la década de los años noventa y se aplica solo en proyectos con una fuerte fundamentación teórica, con claros objetivos de aprendizaje, actividades y componentes de reflexión (Kenworthy-U'ren y Peterson, 2005). El énfasis puede oscilar entre el aprendizaje y el servicio, siendo ambos componentes del modelo; el estudiante adquiere conocimientos prácticos, confronta la teoría y promueve su capacidad de alerta moral (Korpiaho, et al., 2007).

La educación basada en la acción promete una vía de acercamiento a la dicotomía y separación entre el conocimiento académico y la práctica, toda vez que mueve al centro del proceso educativo del administrador la práctica. Los estudiantes aprenden efectivamente no sólo desde su hacer o la experiencia de sus profesores, sino ante todo aprenden sobre la base de la reflexión de sus acciones; 
de igual forma, la manera como se involucra el estudiante lo hace un sujeto activo facilitador del proceso de aprendizaje, en términos de Schön (1983) se refiere al presupuesto pedagógico de la praxis reflexiva y con ello el logro de aprendizaje significativo. No se señala que la orientación sea el logro de curvas de experiencia que permitan mayores eficiencias, sino por el contrario sea posible generar en el profesional la interacción humana y social que soporta su acción, voluntad y condición política.

La educación crítica en Administración se centra en establecer cuestionamientos sobre aquellos presupuestos incuestionables. Esta perspectiva se propone como alternativa de solución al paradigma tradicional dominante, ${ }^{18}$ la cual apunta a revisar los valores y orientaciones de la educación estandarizada actual, debido a que existen para mantener los sistemas económicos fundamentados en la competencia del mercado y los intereses de las personas a cargo de las organizaciones aparentando ser neutrales y libres de valores. El conocimiento de una perspectiva crítica de la Administración contribuye a pensar a través de los valores personales de una forma mucho más explícita;se afirma que la Administración crítica hace un llamado a que los administradores trabajen para transformar las circunstancias en las cuales se encuentran y no solamente se cuestionen los problemas existentes (Learmonth, 2007).

Bajo este esquema, el propósito es que el estudiante aprenda una concepción multidimensional y potencialmente transformadora de la práctica administrativa que pone en el centro las relaciones de poder y la dominación, la eficiencia económica no se discute, pero la ideología de la eficiencia si (Korpiaho, et al., 2007). Para ello el programa se dota de contenidos y métodos soportados desde la teoría crítica; una vez más, se reclama el aprendiz reflexivo que responda y pueda ser movido por las preguntas morales y políticas de sus profesores. El pensamiento crítico aparece como una forma de aprendizaje.

Con todo ello es claro que el administrador, al igual que otros profesionales, se verá enfrentado a los problemas de su quehacer; por lo tanto, deducir de manera antagónica en relación a la validez de una u otra concepción de la educación en administración, sus pretensiones epistemológicas, prácticas, éticas y morales, acompañadas de las distintas modalidades didácticas no es posible. En otras palabras, determinar en términos de si se es blanco o negro, resultaría inadecuado, además de quedar sujeto al riesgo de ser tan ortodoxo como lo que dice evitar.

Pero lo que si ha de comprenderse es que el profesional al igual que la realidad administrativa a la que se ve enfrentado es multidimensional. Además, no ha de olvidarse que las personas, sin importar su profesión, son moralmente responsables y contribuyen a la construcción de una sociedad más justa que privilegie la dignidad, el bienestar y el medio ambiente.

${ }^{18} \mathrm{Al}$ respecto de la necesidad de construir pensamiento crítico, también encontramos voces como Chanlat (1997), Aktouf (2000). 
El reto para las Instituciones de Educación está en reconocer la importancia de la integralidad de los diferentes campos del conocimiento, para lograr una mirada completa en cada una de las disciplinas.

Chanlat (2002, p. 13), considera que entre las grandes transformaciones que ha sufrido la sociedad contemporánea, tres han sido de especial interés: 1) la hegemonía de lo económico, 2) el culto a la empresa, y 3) la influencia creciente del pensamiento gerencial. Respecto al punto número 2, el autor afirma que la empresa se ha convertido en la institución por excelencia, fuente de riqueza, y de cultura, capaz de resolver la mayoría de los problemas, dando relevancia al administrador como actor principal en el proceso.

Para lograr la integración de los campos del conocimiento se debe tener presente que la multidisciplinariedad será una de las bases para la consecución de una formación integral, holística, capaz de conocer e interpretar las realidades de cada una de las disciplinas; es una mirada del mundo desde varias perspectivas, todas válidas; es claro que el reduccionismo limita las formas de pensar y comprender el mundo, "lo que reclamamos no es abolir la disciplina, sino el reconocimiento del derecho e incluso del deber de circular, la valorización de los tránsitos, el levantamiento de barreras para la asociación con otras especies: sociólogos, economistas, biólogos, filósofos, etc. La libertad de tejer redes a voluntad" (Limoges, citado por Chanlat, 2002, p. 65).

\section{Conclusión}

La apertura de pensamiento y de las fronteras de las disciplinas, permitirá beneficios tales como: hacer del proceso de formación del administrador, un proceso libre, generador de pensamiento crítico, seres humanos sensibles, con capacidad para trabajar con otros diferentes a él, con mente abierta, y con capacidad de interpretación de los diferentes fenómenos sociales, organizacionales, con los cuales se deben enfrentar; otro beneficio tiene que ver con la construcción del conocimiento, que aporte a la construcción de la sociedad; Como lo afirma Hayek, premio Nobel de economía: "Nadie puede ser un gran economista si solamente es un economista"; "un economista que sólo sea un economista tiene todas las posibilidades de llegar a ser dañino e, incluso, realmente peligroso"; algunos califican este fenómeno de la multidisciplinariedad como una híbrido dañino para las disciplinas, porque permite el tránsito de unos y otros sin profundidad en los diferentes temas de interés para el fortalecimiento de las mismas. A lo cual Chanlat comenta: "Los híbridos son, pues, creadores que ven de otra manera e innovan en los márgenes, nunca en el centro que siempre está ocupado por los guardianes de la ortodoxia" (2002, p. 67).

Todo lo anterior apoya la postura de Mintzberg (2004) y Aktouf (2002), al proponer que en lugar de una visión instrumental, reduccionista y funcionalista de la Administración se debe ir a una centrada en lo humano y el desarrollo social. Es necesario desde la educación cambiar contenidos, metodologías, prácticas; educar más que entrenar (Mintzberg, 2004, p. 384), y desde el pregrado educar en las disciplinas que nutren y sirven de raíz a la Administración (ciencias sociales y 
matemáticas) (2004, p. 385), "despertar la sensibilidad personal, ofrecerle a los estudiantes mayor cultura" (Aktouf, 2002, p. 62).

\section{Fuentes DE Consulta}

- AKTOUF, O. (2000). La Administración entre Tradición y Renovación,Cali: Univalle

$-$ Universidad Eafit.

(2002). Administración y pedagogía,Medellín: Fondo Editorial

- ARMSTRONG, S. \&Fukami, C. (2010). Self-Assessment of knowledge: a cognitive learning or affective measure? Perspectives from the management learning and education community. Academy of Management Learning \& Education, 9, 2, 335-341.

- BARRERA, E. y Sanclemente, J. (2008). Una perspectiva genealógica y crítica para repensar la Administración. RevistaUniversidad Eafit, 44, 152.

- BEATTY, J. and Leigh, J. (2010). Taking stock of management education: comparison of three management education journals. Journal of Management Education 34, 3, 367-392.

- BENEITONE, P. Esquetini, C. González, J. Maletá, M. Siufi, G. y Wagenaar, R. (editores) (2007). TuningAmérica Latina. Reflexiones y perspectivas de la educación superior en América Latina,Bilbao: Publicaciones Universidad de Deusto.

- BUCHELLI, G. \& Marín, J. (2009).Transposición Didáctica: bases para repensar la enseñanza de una disciplina científica. I parte.Revista Académica e Institucional, Páginas de la UCPR, 85, 17-38.

- CAMILLONI, A. Davini, M. Edelstein, G. Litwin, E. Suoto, M. y Barco, S. (1996) Corrientes didácticas contemporáneas, Buenos Aires: Paidós.

- CONTRERAS, D. (1990). La didáctica y los procesos de enseñanza aprendizaje. En: "Enseñanza, Currículum y Profesorado. Introducción a la didáctica", Madrid: Akal.

- CHANLAT, A. y Bédard, R. (1997) La administración, una cuestión de palabra. Tecnología Administrativa.U. de A. 6, 25, 79-94.

- CHANLAT, J. F. (2002). Ciencias Sociales y Administración. En defensa de una antropología general,Medellín: Fondo Editorial Universidad Eafit.

- DAY, G. y Schoemaker, P. (2001).Gerencia de tecnologías emergentes, Buenos Aires: Editorial Vergara/Business.

- DE MATTOS (1983). Compendio de didáctica general. Capitulo V. didáctica, su objeto y sus problemas, Buenos Aires: Editorial Kapeluz.

- DELORS, J. (1998). La educación encierra un tesoro. Informe Unesco de la Comisión Internacional sobre la Educación para el Siglo XXI, Ediciones UNESCO. Compendio. 
- FERMOSO, P. (1985). Teoría de la educación: una interpretación antropológica, Barcelona: CEAC.

- FLORES Ochoa, R. (1994). Hacia una pedagogía del conocimiento,Bogotá: Mc Graw-Hill.

- GRISALES-Franco, L. M. y González-Agudelo, E. M. (2009). El saber sabio y el saber enseñado: un problema para la didáctica universitaria. Educación y Educadores, 12, 2, 77-86.

- KENWORTHY-U'ren, A.L. \& Peterson, T.O. (2005). Service-learning and management education: Introducing the "WE CARE" approach. Academy of Management Learning \& Education, 4, 3, 272-275.

- KORPIAHO, K. Päiviö, H. y Räsänen, K. (2007). Anglo-American forms of management education: A practice-theoretical perspective. Scandinavian Journal of Management. 23, 1, 36 - 65.

- LEARMONTH, M. (2007). Critical Management Education in Action: Personal Tales of Management UnlearningAcademy of Management.Learning\&Education, 6, 1, 109-113.

- LÓPEZ Gallego, F. (1998). Educación en Administración y modas administrativas en Colombia.Revista Universidad Eafit, 109, 59 - 88.

- LUCIO, R. A. (1989): Educación y pedagogía, enseñanza y didáctica: diferencias y relaciones. Recuperado 24, 6, 2010de la fuente: http://www.fum.edu.co/snies/inst/programas/educacionPreescolar/doc_dimp lom/Documentos/Educaci\%C3\%B3n\%20y\%20pedagogia\%20ricardo\%20luc io.pdf. También se puede encontrar publicado en: Lucio, A. (1989) Educación y pedagogía, enseñanza y didáctica: diferencias $y$ relaciones.Revista Universidad de la Salle, 17, 35-46.

- MINTZBERG, H. (2004). Managers notMBA's, San Francisco:BarrettKoehler.

dirección (2005). Directivos, no MBA's: una visión crítica de la de empresas y la formación empresarial, Barcelona: Ediciones Deusto.

- PAPALIA, D. Wendkos, S. Duskin, R. y Gross, D. (2005).Desarrollo Humano, Madrid:McGraw.

- PHAN, P. Siegel, D. \& Wright, M. (2009).New developments in technology management education: background issues, program initiatives, and a research agenda.Academy of Management Learning\&Education, 8, 3, 324336.

- PATIÑO y Rojas (2009). Subjetividad y subjetivación de las prácticas pedagógicas en la universidad. Revista Educación y Educadores, 12, 1, 93105.

- ROZO Sandoval, C. (2008). Contextos y pretextos sobre la pedagogía,Bogotá: Universidad Pedagógica Nacional. 
- SERNA, G. H. (2001). Índices de gestión. Cómo diseñar un sistema integral de medición de gestión, Bogotá: $3 \mathrm{R}$ Editores.

- SHÖN, Donald A. (1983). The reflective practitioner: How professionals think in action,London: Temple Smith.

- SHULMAN, Lee S. (2005). Signature pedagogies in the professions. Daedalus. 134, 3, 52-59.

- TAYLOR, J. \& Machado, M. (2006). Higher education leadership and management: from conflict to interdependence through strategic planning. Tertiary Education and Management 12, 137-160.

- VANCE, C. Groves, K. Yongsun, P. \&Klindler, H. (2007). Understanding and measuring linear-nonlinear thinking style for enhanced management education and professional Practice.Academy of Management Learning\&Education, 6, 2, 167-185.

- VASCO Uribe, C. E. (1990). Reflexiones sobre pedagogía y didáctica, Bogotá: Ministerio de Educación Nacional.

- WEBER M. (2004).La ética protestante y el espíritu del capitalismo, México: Premia Editores 